\title{
A probabilistic framework to infer connectivity from function: a study of change detection and adaptation Nabil Bouaouli* and Sophie Denève
}

\author{
Address: Group of Neural Theory, Ecole Normale Supérieure, Paris, France \\ Email: Nabil Bouaouli* - nabil.bouaouli@ens.fr \\ * Corresponding author
}

from Eighteenth Annual Computational Neuroscience Meeting: CNS*2009

Berlin, Germany. 18-23 July 2009

Published: 13 July 2009

BMC Neuroscience 2009, I0(SuppI I):OI8 doi:I0.I I86/I47I-2202-I0-SI-OI8

This abstract is available from: http://www.biomedcentral.com/I47I-2202/I0/SI/OI8

(C) 2009 Bouaouli and Denève; licensee BioMed Central Ltd.

A marked feature of sensory neurons is their propensity to respond more strongly to transient rather than to steady stimuli. While a variety of mechanisms, like short-term depression and spike rate adaptation, boost responses to higher temporal frequencies, change detection as a key aspect of sensory processing remains largely unexplored. To test this hypothesis, we use a novel probabilistic framework and show quantitatively the importance of feed-forward inhibition in detecting sudden "appearance" of stimuli. The model accounts for some coding strategies in the early visual system and suggests a neuronal microcircuit that could achieve this function.

Assuming Markov dynamics of real world stimuli and Poisson input spike trains, we derive an ideal observer that computes on-line the probability of a sudden stimulus appearance by reading out changes in input firing rates. The resulting integration dynamics resembles that of real neurons. It predicts a biphasic, nonlinear synaptic integration consisting of a fast, transient excitation followed by a slower long lasting inhibition. The same stimulus exciting the cell at short delays inhibits it at longer ones. However, the properties of this integration (i.e. the strength and time constants of excitation and inhibition) depend on the temporal statistics of stimuli and the reliability of input spike trains.

We then explore what this general framework implies for neural coding in terms of temporal receptive fields (tRFs), contrast adaptation and spike-time precision in early vis- ual areas. We found that our model reproduces the firing statistics of the retina and LGN in response to time- (and contrast-) varying stimuli. Particularly, sharp peaks in the resulting PSTH are well described. The predicted tRFs resemble those of "ON" and "OFF" retinal ganglion cells and LGN cells. Interestingly, as a result of model nonlinearities, their shape adapts to input contrast as experimentally reported. Integration dominates at low contrasts, while temporal derivation occurs at higher ones. Moreover, in agreement with experimental data, the bursts of spikes obtained are preceded by strong inhibition while isolated spikes are not.

As a biophysical implementation of this adaptive change detection model, we suggest a microcircuit that is commonly observed in many sensory areas. Namely, a pyramidal neuron targeting another pyramidal neuron both directly with a depressing synapse and indirectly with a facilitating synapse through an inhibitory interneuron. Finally, we show how this inhibition modulates the performance in detection and discuss its more general role in sensory processing over different timescales. 